\title{
ORIGINAL
}

1MÉDICO PEDIATRA. HOSPITAL MATERNO INFANTIL BOLIVIANO JAPONÉS. TRINIDAD - BENI - BOLIVIA.

2 2MÉDICO RESIDENTE II NIVEL. HOSPITAL MATERNO INFANTIL BOLIVIANO JAPONÉS. TRINIDAD - BENI - BOLIVIA

Correspondencia a: Correo electrónico: migceles@hotmail.com

Palabras clave: Germenes, in fecciones, pediatria.

Keywords: Germs, Infections, pediatrics.

Procedencia y arbitraje: no comisionado, sometido a arbitraje externo.

Recibido para publicación: 05 de Abril del 2017 Aceptado para publicación: 25 de Junio del 2017

Citar como: Rev Cient Cienc Med 2017;20(1): 20- 25

\section{GÉRMENES MÁS FRECUENTES EN INFECCIONES ASOCIADAS A LA ATENCIÓN EN SALUD EN LA UTI PEDIÁTRICA}

\author{
FREQUENT GERMS IN INFECTIONS ASSOCIATED WITH HEALTH CARE IN \\ UCI PEDIATRIC
}

\author{
Dr. Miguel Céspedes Lesczinsky', Dra. Zurama Velasco Abularach²
}

\section{RESUMEN}

Las infecciones asociadas a la atención en salud representan un grave problema para los pacientes atendidos en hospitales, más aun tratándose de niños en la Unidad de Terapia Intensiva, por tal motivo realizamos este estudio de tipo descriptivo retrospectivo y de corte transversal durante un período de 4 años (2012 - 2015) con el fin de conocer los gérmenes más frecuentes y su sensibilidad antibiótica en la Unidad de Terapia Intensiva Pediátrica del Hospital Materno Infantil Boliviano Japonés de la ciudad de Trinidad, en el mismo que ingresaron aquellos pacientes admitidos en la unidad con cultivos positivos de distintas localizaciones. Los resultados obtenidos muestran que los ${ }^{3}$ gérmenes más frecuentes en la unidad son Klebsiella, Enterobacter y Burkholderia, siendo esta última la causa de mayor mortalidad entre los pacientes cuyos cultivos fueron positivos, los hemocultivos obtuvieron mayor porcentaje de positivos, Candida albicans fue más frecuente en urocultivos y E. coli en coprocultivos. Burkholderia sólo se muestra sensible a Meropenem y Cotrimoxazol, Enterobacter a Imipenem, Amikacina y Ciprofloxacina, Klebsiella a Imipenem, Meropenem y Amikacina. Llamativa la resistencia de todos los microorganismos a Gentamicina y Cefalosporinas. Dejamos en claro que es importante la puesta en marcha de un Comité de Infecciones Hospitalarias, a fin de poder aunar esfuerzos y directrices que lleven a la reducción de la incidencia y prevalencia de estos gérmenes en la unidad, tomando en cuenta que son pocas las alternativas de tratamiento antibiótico con las que contamos.

\section{ABSTRACT}

Infections associated with health care represent a serious problem for patients treated in hospitals, even more so in the case of children in the Intensive Care Unit, for this reason we performed this descriptive, retrospective and cross-sectional study during a period of 4 years (20I2-20I5) in order to know the most frequent germs and their antibiotic sensitivity in the Pediatric Intensive Care Unit of the Hospital Materno Infantil Boliviano Japonés of the city of Trinidad, in the same one that admitted patients admitted in the unit with Positive cultures from different locations. The results obtained show that the 3 most frequent germs in the unit are Klebsiella, Enterobacter and Burkholderia, the latter being the cause of higher mortality among patients whose cultures were positive, blood cultures obtained a higher percentage of positive, Candida albicans was more frequent in Urocultures and E. coli in co-cultures. Burkholderia is only sensitive to Meropenem and Cotrimoxazole, Enterobacter to Imipenem, Amikacin and Ciprofloxacin, Klebsiella to Imipenem, Meropenem and Amikacin. The resistance of all microorganisms to Gentamicin and Cephalosporins is striking. We made it clear that it is important to set up a Hospital Infection Committee so as to be able to combine efforts and guidelines that lead to a reduction in the incidence and prevalence of these germs in the unit, taking into account that there are few alternatives Of antibiotic treatment with which we count.

\section{INTRODUCCIÓN}

a infección asociada a la atención en salud (IAAS) se define clásicamente como aquella que aparece 48 horas luego del ingreso, durante la hospitalización o hasta 72 horas después del alta. El Centro para el Control y Prevención de Enfermedades de Atlanta (Center of Desea- se Control, $C D C$ ) define a la IAAS como aquella que no está presente o incubándose en el momento del ingreso al hospital, que se manifiesta clínicamente o es descubierta durante un procedimiento quirúrgico, endoscópico u otro procedimientos o pruebas diagnósticas; o que se base en criterio clínico. 
Se incluyen las infecciones que por su tiempo de incubación se manifiestan luego del alta del paciente y se relacionan con los procedimientos o actividades hospitalarias ${ }^{1}$.

La IAAS es una causa importante de morbimortalidad en pacientes atendidos en los hospitales, con costos altamente significativos para el paciente y el sistema de salud. Los pacientes pediátricos que poseen mayor riesgo de presentar estas infecciones, son aquellos atendidos en la unidad de terapia intensiva (UTI), oncológicos, inmunodeficientes, quirúrgicos sobre todo cardiacos o aquellos que son sometidos a métodos diagnósticos y tratamientos invasivos ${ }^{2}$. La bacteriemia relacionada con el catéter venoso central suele ser la primera causa de IAAS en pacientes pediátricos críticos, seguida de la neumonía, asociada al ventilador mecánico, y la infección del tracto urinario secundaria al uso de sonda vesical ${ }^{3}$. A las dos primeras se les atribuye el $60 \%$ de mortalidad ${ }^{4}$. La tasa de las IAAS en la unidad de terapia intensiva pediátrica (UTI-P) es de 18,7\%5. La prevalencia de las IAAS difieren entre regiones, así en EEUU se reporta entre 5 a 7\%, en Latinoamérica tiene una prevalencia que oscila entre 7 a $40 \%^{2}$. La OMS reporta una prevalencia de 5,7\% y $19,1 \%$ en los hospitales de mediana y alta complejidad ${ }^{4}$.

Tomando en cuenta estos datos es que se hizo necesaria la aplicación de acciones que sean destinadas a la prevención de las IAAS, como ser: estricto lavado de manos, asepsia, antisepsia, esterilización y desinfección, uso de guantes, barbijos, batas estériles durante procedimientos invasivos, uso racional de antibióticos, nutrición y esquema de vacunación adecuado6. Se ha demostrado ampliamente que la existencia de un Comité de Infecciones Nosocomiales que establezca programas de vigilancia, control y prevención de enfermedades, constituye un pilar fundamental para la prevención de estas IAAS 2,6 .

El manejo inicial de las IAAS se hizo de acuerdo a la epidemiología propia del hospital y el perfil de resistencia de los germenes más frecuentes ${ }^{6}$, de ahí la importancia de este estudio, tomando en cuenta la falta de datos en la UTI-P del hospital, realizamos el mismo con la intención de conocer la realidad en cuanto a gérmenes más frecuentes y resistencia que presentan a los antibióticos más utilizados, para la elaboración de estrategias que estén destinadas a disminuir la prevalencia de las IAAS.

\section{MATERIALES Y MÉTODOS}

Se realizó un estudio retrospectivo de tipo descriptivo y corte transversal en la UTI-P del Hospital Materno Infantil Boliviano Japonés de la ciudad de Trinidad, durante un período de 4 años (enero 2012 - diciembre 2015). La recolección de datos se realizó mediante la revisión de las historias clínicas de los pacientes cuyo resultado de cultivos fue positivo, se clasificó como tal informando crecimiento de algún germen. Para la recolección de los mencionados datos se elaboró una planilla.

Los cultivos que se tomaron en cuenta procedían de muestras de sangre, orina, heces fecales, secreciones bronquiales así como elementos externos insertados en pacientes graves, como tubo endotraqueal (TET), vía central o sonda vesical.

Se consideró para el presente estudio como infección nosocomial en la UTI-P a aquellos pacientes cuyo cultivo resultara positivo luego de 48 horas del ingreso a la unidad.

Se establecieron como variables a tener en cuenta las siguientes: edad, sexo, gérmenes más frecuentes, porcentaje de cultivos positivos, antibiograma y mortalidad.

Se procedió a la tabulación y los resultados se expresan en valores absolutos y porcentuales.

\section{RESULTADOS}

Durante el período de estudio fueron ingresados a la UTI-P un total de 451 pacientes, siendo la gestión con mayor número de ingresos el año 2012 con 159 pacientes (ver tabla 1), el sexo predominante fue el femenino con $60 \%$ (ver tabla 2), el grupo etario con mayor número de pacientes fue de 1 mes a 2 años con $69 \%$ del total (ver tabla 3 ).

Existieron 109 fallecidos, la gestión con mayor número de óbitos fue el año 2013 con 38 casos (ver tabla 1), siendo el sexo masculino el más comprometido con $56 \%$ de casos (ver tabla 4) y el grupo etario de 1 mes a 2 años reporto una de mortalidad de 64\% (ver tabla 5).

En cuanto a los resultados de los cultivos, del total de pacientes ingresados en la UTI-P, se encontraron 115 cultivos positivos de los cuales los hemocultivos representan el mayor número 37 (ver tabla 6), seguido de cultivos de punta de catéter y punta de TET. Los gérmenes aislados con mayor frecuencia en el global de los cultivos fueron Klebsiella con 26 cultivos positivos, seguidos de Enterobacter y Burkholderia. (ver tabla 6). Cabe mencionar que el germen más frecuente en coprocultivo fue E. Coli, y C. albicans el más común en urocultivos (ver tabla 6).

Respecto al día de aparición del cultivo positivo, fue más frecuente entre el 3er y 8 vo día (ver gráfico 1). Del global de cultivos positivos, los 3 gérmenes más frecuentes representaronn un total de 61 cultivos, considerando que el porcentaje de pacientes fallecidos fue mayor en aquellos en cuyo cultivo se aisló Burkholderia, 93\% de mortalidad, 
seguido de Enterobacter y Klebsiella (ver tabla 7). Tomando en cuenta la sensibilidad del antibiograma en estos 3 gérmenes sólo Meropenem logra buenos resultados terapéuticos in vitro en todos ellos, seguido de Imipenem y amikacina. Es muy importante hacer notar que Burkholderia sólo fue sensible a Meropenem y Cotrimoxazol. Considerando el resultado de resistencia en el antibiograma, el antimicrobiano

\begin{tabular}{|llll|}
\hline Tabla 1 & & & \\
\hline AÑO & INGRESOS & FALLECIDOS & $\%$ \\
\hline 2012 & 159 & 19 & $12 \%$ \\
\hline 2013 & 87 & 38 & $44 \%$ \\
\hline 2014 & 98 & 25 & $26 \%$ \\
\hline 2015 & 107 & 27 & $25 \%$ \\
\hline TOTAL & 451 & 109 & $24 \%$ \\
\hline
\end{tabular}

\begin{tabular}{|lllll|}
\hline $\begin{array}{l}\text { Tabla } 3 \\
\text { AÑO }\end{array}$ & $1 \mathrm{~m}-2 \mathrm{a}$ & $2 \mathrm{a}-5$ & $>5 \mathrm{a}$ & TOTAL \\
\hline 2012 & 123 & 16 & 20 & 159 \\
\hline 2013 & 60 & 10 & 17 & 87 \\
\hline 2014 & 62 & 17 & 19 & 98 \\
\hline 2015 & 66 & 16 & 25 & 107 \\
\hline TOTAL & 311 & 59 & 81 & 451 \\
\hline$\%$ & $69 \%$ & $13 \%$ & $18 \%$ & $100 \%$ \\
\hline
\end{tabular}

\begin{tabular}{|lllll|}
\hline $\begin{array}{l}\text { Tabla } 5 \\
\text { AÑ }\end{array}$ & $1 \mathrm{~m}-2 \mathrm{a}$ & $2 \mathrm{a}-5 \mathrm{a}$ & $>5 \mathrm{a}$ & TOTAL \\
\hline 2012 & 13 & 2 & 4 & 19 \\
\hline 2013 & 20 & 6 & 12 & 38 \\
\hline 2014 & 19 & 4 & 2 & 25 \\
\hline 2015 & 18 & 1 & 7 & 26 \\
\hline TOTAL & 70 & 13 & 26 & 109 \\
\hline$\%$ & $64 \%$ & $12 \%$ & $24 \%$ & $100 \%$ \\
\hline
\end{tabular}

con la mayor resistencia fue Gentamicina. Es importante resaltar que los resultados de resistencia en los antibiogramas fueron considerables para Cefotaxima, Ceftazidima y Amoxicilina/ Ácido Clavulánico (ver tabla 8). En aquellos pacientes cuyo resultado de cultivo fue positivo, 46 estuvieron en asistencia respiratoria mecánica y 38 recibieron aminas endovenosas (ver tabla 9).

\begin{tabular}{|llll|}
\hline Tabla 2 & & & \\
\hline AÑO & FEMENINOS & MASCULINOS & TOTAL \\
\hline 2012 & $\mathbf{8 7}$ & $\mathbf{7 2}$ & $\mathbf{1 5 9}$ \\
\hline $\mathbf{2 0 1 3}$ & $\mathbf{5 6}$ & $\mathbf{3 1}$ & $\mathbf{8 7}$ \\
\hline $\mathbf{2 0 1 4}$ & $\mathbf{5 9}$ & $\mathbf{3 9}$ & $\mathbf{9 8}$ \\
\hline $\mathbf{2 0 1 5}$ & $\mathbf{6 2}$ & $\mathbf{4 5}$ & $\mathbf{1 0 7}$ \\
\hline TOTAL & $\mathbf{2 6 4}$ & $\mathbf{1 8 7}$ & $\mathbf{4 5 1}$ \\
\hline \% & $\mathbf{6 0 \%}$ & $\mathbf{4 0 \%}$ & $\mathbf{1 0 0 \%}$ \\
\hline Tabla 4 & & & \\
\hline AÑO & FEMENINOS & MASCULINOS & TOTAL \\
\hline 2012 & 11 & 8 & 19 \\
\hline 2013 & 14 & 24 & 38 \\
\hline 2014 & 11 & 14 & 25 \\
\hline 2015 & 11 & 15 & 26 \\
\hline TOTAL & 48 & 61 & 109 \\
\hline$\%$ & $44 \%$ & $56 \%$ & $100 \%$ \\
\hline
\end{tabular}

Grafico 1

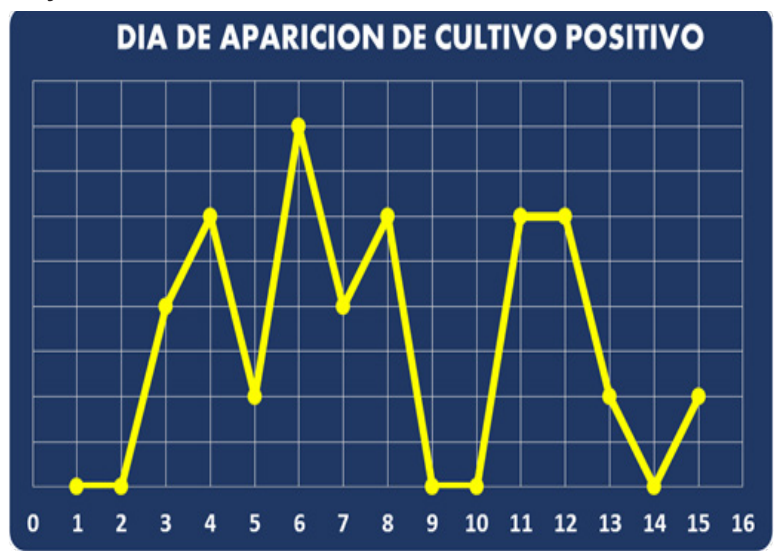

\begin{tabular}{|c|c|c|c|c|c|c|}
\hline \multicolumn{7}{|l|}{ Tabla 7} \\
\hline \multicolumn{7}{|c|}{ GERMEN Y MORTALIDAD } \\
\hline GERMEN & VIV & & FAL & & TO & \\
\hline Burkholderia & 1 & $7 \%$ & 14 & $93 \%$ & 15 & $25 \%$ \\
\hline Enterobacter & 11 & $55 \%$ & 9 & $45 \%$ & 20 & $33 \%$ \\
\hline \multirow[t]{2}{*}{ Klebsiella } & 20 & $77 \%$ & 6 & $23 \%$ & 26 & $42 \%$ \\
\hline & & & & & 61 & $100 \%$ \\
\hline
\end{tabular}




\begin{tabular}{|c|c|c|c|c|c|c|c|c|c|}
\hline GERMEn & HEMOCULTIVO & COPROCULTIVO & UROCULTIVO & $\begin{array}{l}\text { SECRECION } \\
\text { DE TET }\end{array}$ & $\begin{array}{l}\text { PUNTO } \\
\text { DE TET }\end{array}$ & $\begin{array}{l}\text { PUNTA } \\
\text { DE } \\
\text { CATETER }\end{array}$ & LCR & OTROS & TOTAL \\
\hline KLEPSIELA & 11 & 2 & 1 & 2 & 4 & 5 & 0 & 1 & 26 \\
\hline ENTROBACTER & 8 & 2 & 1 & 2 & 3 & 2 & 1 & 1 & 20 \\
\hline BURKHOLDERIA & 7 & 0 & 0 & 2 & 3 & 3 & 0 & 0 & 15 \\
\hline STAPHILOCOCCUS & 2 & 0 & 0 & 1 & 0 & 1 & 1 & 5 & 10 \\
\hline CANDIDA & 1 & 1 & 4 & 1 & 0 & 2 & 0 & 0 & 9 \\
\hline PSEUDOMONA & 1 & 1 & 0 & 2 & 1 & 1 & 0 & 2 & 8 \\
\hline E.COLI & 1 & 4 & 1 & 0 & 1 & 1 & 0 & 0 & 8 \\
\hline ACINETOBACTER & 1 & 1 & 0 & 2 & 0 & 0 & 0 & 0 & 7 \\
\hline STENOTROPOMONA & 0 & 1 & 1 & 1 & 2 & 0 & 0 & 1 & 6 \\
\hline CITROBACTER & 1 & 0 & 0 & 0 & 0 & 1 & 0 & 1 & 3 \\
\hline STREPTOCOCCUS & 0 & 0 & 0 & 0 & 1 & 0 & 1 & 0 & 2 \\
\hline SERRATIA & 1 & 0 & 0 & 0 & 0 & 0 & 0 & 0 & 1 \\
\hline TOTAL & 37 & 12 & 8 & 13 & 15 & 16 & 3 & 11 & 115 \\
\hline
\end{tabular}

\section{DISCUSIÓN}

Reiteradamente se reporta una creciente gravedad del paciente infectado en la UTI, así como una creciente dificultad en su manejo, debido a la alta frecuencia de infecciones por gérmenes resistentes y sus reducidas opciones terapéuticas.

Además la UTI-P constituye el epicentro de diseminación de gérmenes resistentes a otras áreas de un hospital, convirtiéndose los pacientes y el medio ambiente en los principales reservorios $^{7}$. Se determinaron algunos factores que predisponen a la presencia de IAAS. Los relacionados al huésped como ser: menores de 2 años, inmunodeficiencias, antecedente de prematurez, enfermedad de base severa, neutropenia, desnutrición. Otros relacionados con la asistencia: días de permanencia en la unidad, días de permanencia del catéter o de la asistencia respiratoria mecánica (ARM), uso de nutrición parenteral. Así como factores propios de los gérmenes, por ejemplo estafilococo coagulasa negativo y K. pneumoniae tienen la capacidad de formar una glicoproteína que forma el slime alrededor de las colonias que determina mayor adherencia a cuerpos extraños y protege de las defensas del huésped y de los antibióticos ${ }^{8,9}$ En este estudio al igual que otros demostramos que las bacterias son los principales gérmenes que infectan a los pacientes hospitalizados en UTI-P, quedando en escasa proporción los hon- gos y no haciéndose investigación etiológica para virus. Los gérmenes Gram negativos aerobios forman una clase predominante de patógenos resistentes dentro de las infecciones nosocomiales en UTI-P ${ }^{10}$. En el presente trabajo no se establecieron diferencias entre gérmenes que colonizaron o infectaron a los pacientes.

Del total de ingresos, el sexo femenino fue el predominante a diferencia de los distintos reportes en la literatura médica sobre infecciones nosocomiales en UTI en el mundo, en los cuales el sexo masculino es el predominante, los fallecidos representan $24,1 \%$, algo similar a lo obtenido en el trabajo de Jover Saenz ${ }^{11}$, el porcentaje de fallecidos durante la gestión 2013 fue el más elevado, duplicando el de otros años, sin embargo desconocemos las razones por la cual esta situación se presentó.

El grupo etario con el mayor número de ingresos y fallecidos fue el comprendido entre 1 mes a 2 años, que representa el $64 \%$ del total de las defunciones, algo similar a otros estudios ${ }^{2,12}$, esto se puede explicar por varias causas, entre las que podríamos mencionar: un sistema inmunitario inmaduro que lleva a una pobre respuesta contra la infección, el estado nutricional deficiente, una inmunización incompleta, la falta de una adecuada lactancia materna entre otros.

Tomando en cuenta la mortalidad el sexo masculino tuvo mayor predominio con un $56 \%$. 
En el actual estudio los gérmenes más frecuentes en los cultivos de pacientes con infecciones nosocomiales de la UTI-P fueron KlebsieIla, Enterobacter y Burkholderia, este resultado contrasta con otros en los que Pseudomona es el germen más frecuente al igual que en los trabajos de Luani Badel et al y Briceño et $\mathrm{al}^{1,13}$, o S. aureus como lo reporta Solano Chinchilla ${ }^{14}$. En el trabajo de Salazar Gonzáles et al, Klebsiella sería el 3er germen más frecuente en las infecciones en UTI ${ }^{10}$, Rodríguez Camacho y col reportan en su artículo que cuando se observa infecciones nosocomiales de manera global, E. coli sería el germen más frecuente, siendo Klebsiella nuevamente el 3ero en frecuencia ${ }^{7}$. En este estudio Klebsiella fue aislado con mayor frecuencia en hemocultivos, resultados similares a los obtenidos por Salazar Gonzáles ${ }^{10}$.

Candida fue más frecuente en urocultivos, tal y como lo demuestran otros trabajos similares ${ }^{10}$.

Es muy llamativo que gérmenes como Burkholderia estén entre los más frecuentes en las infecciones nosocomiales de la UTI, puesto que en los reportes de la literatura, son otros los gérmenes que con mayor frecuencia están involucrados en este tipo de infecciones, es más que preocupante que en esta unidad, un germen multirresistente sea uno de los más significativos, a sabiendas que es un bacilo gram negativo aerobio, productor de catalasa, no fermentador y que prolifera en condiciones nutricionales mínimas, además que puede sobrevivir en soluciones detergentes y desinfectantes, sistemas de ventilación, soluciones y dispositivos intravenosos, cuyo espectro antibiótico de sensibilidad se limita a Meropenem, Cotrimoxazol, Piperazilina/Tazobactam tal como demuestra el trabajo de Cardoso et al. Asimismo es importante hacer notar que en este estudio la mortalidad asociada esta bacteria fue de $93 \%$ de los pacientes con cultivo positivo a la misma, lo que debiera llevarnos a tomar medidas estrictas para disminuir su incidencia en las infecciones de la UTI.

Enterobacter es otro de los gérmenes frecuentes en el presente estudio, algo que es totalmente distinto a otros reportes, Rodríguez Camacho menciona como el menos frecuente en infecciones nosocomiales globales ${ }^{7}$, Quintanilla Chanez en un trabajo nacional reporta un $6 \%$ de infecciones por esta bacteria en su mayoría asociadas a ARM ${ }^{16}$, Luna et al reportan un porcentaje similar ${ }^{17}$. En la presente investi- gación se reporta una mortalidad de $45 \%$ por este germen siendo la segunda causa de óbitos por infecciones en la UTI, haciendo énfasis en que la sensibilidad antibiótica se limita a Imipenem, Amikacina y Ciprofloxacina, algo similar a lo reportado por Briceño y Suárez en Venezuela y Quintanilla Chanez en Bolivia. Enterobacter en otros análisis se reporta como causa menos frecuente de infecciones en la UTI1, 14,16, en este trabajo ocupa el segundo lugar en los cultivos positivos.

Klebsiella aunque es el germen más frecuente en la UTI, es la tercera cuando analizamos la mortalidad asociada a infecciones nosocomiales y aunque el porcentaje es menor respecto de las anteriores, el espectro de sensibilidad antibiótica también es limitado a Imipenem, Meropenenm y Amikacina.

Haciendo notar la sensibilidad de los tres gérmenes más frecuentes en la UTI-P, ninguno de ellos responde a Gentamicina, Cefotaxima, Ceftazidima. Enterobacter y Klebsiella son resistentes a Amoxicilina/Ac Clavulánico, Klebsiella muestra resistencia a Ciprofloxacina y Cotrimoxazol. Estos hallazgos concuerdan con lo reportado en la literatura ${ }^{18}$ y debe llevarnos a tomar acciones para evitar la proliferación de estos en la unidad. Entre estas acciones, la higiene de manos es importante, que como práctica preventiva, goza de mayor difusión y eficacia, sin embargo una de las barreras para su aplicación es el conocimiento de los profesionales en salud sobre su eficacia ${ }^{19}$. Luego de la realización del presente estudio comenzaremos a implementar en la UTI-P los protocolos de bacteriemia cero y neumonía cero, cuyos resultados serán monitorizados a fin de poder mejorar la atención en salud.

Al revisar la literatura médica notamos que los reportes difieren unos de otros cuando de prevalencia de gérmenes se refiere, de ahí la importancia de poder establecer la propia casuística de las infecciones y sensibilidad antibiótica en las UTI de cada hospital, para tener una base de la cual tomar un adecuado tratamiento empírico inicial, el mismo que será modificado luego, de acuerdo a germen aislado y sensibilidad conocida. 


\section{REFERENCIAS}

1. Badell LL, Losa PD, Ávila RM, Barletta CJ, HernándezMS, GómezMA. CARACTERIZACIÓN DE LA INFECCIÓN NOSOCOMIAL EN UNA UNIDAD DE CUIDADOS INTENSIVOS PEDIÁTRICOS 2014. Disponible en: $h$ ttp://scielo. sld.cu/pdf/ms/v12n3/ms02312.pdf

2. Rodríguez M, Duarte A, Alfieri P, Basualdo W. INFECCIONES INTRAHOSPITALARIAS EN UNA UNIDAD DE CUIDADOS INTENSIVOS PEDIÁTRICOS. Análisis de tres años de vigilancia (2006-2008). Pediatr. (Asunción). 2010; 37 (1); 23-29.

3. Jordán GI, Bustinza AA, Concha TJ, Gil AJ,Vicente J, Téllez GC. ESTUDIO MULTICÉNTRICO NACIONAL SOBRE LA INFECCIÓN NOSOCOMIAL EN LA UCIP. An Pediatr (Barc) 2014. 80 (1); 28-33.

4. Londoño J, Macias I, Ochoa F. FACTORES DE RIESGO ASOCIADOS A INFECCIONES POR BACTERIAS MULTIRRESISTENTES DERIVADAS DE LA ATENCIÓN EN SALUD EN UNA INSTITUCIÓN HOSPITALARIA DE LA CIUDAD DE MEDELLÍN 2011-2014. Infectio. 2016; 20 (2); 77-83.

5. Lombardo $A E$, Hernández $O H$, Orozco $H$, Soto E. VIGILANCIA EPIDEMILÓGICA EN EL SERVICIO DE TERAPIA INTENSIVA DE UN HOSPITAL PEDIÁTRICO DE TERCER NIVEL. Acta Pediatr Mex 2011. 32 (4); 209-12.

6. Salazar CV. INFECCIONES INTRAHOSPITALARIAS. Rev Bol Soc Ped 2012. 51 (3); 187-90.

7. Rodríguez CE, Díaz GB. INFECCIONES/ COLONIZACIONES POR GÉRMENES MULTIRESISTENTES. Galicia Clin 2014. 75 (1); 17-21.

8. Bustinza AA, Solana GM, Botrán PM, Padilla OB. INFECCIÓN NOSOCOMIAL. López-Herce J. Manual de Cuidados Intensivos Pediátricos 2013. 4ta ed. Madrid; 310-322. Publimed

9. Fernández $G$, Turina $D$. INFECCIÓN RELACIONADA AL CATÉTER. Farias J, Neira $P$, Koch E, Nieva A. Guías de Terapia intensiva Pediátrica. Bs As. Corpus. 2015. 308-15.

10. Salazar GT, Morejón CD, Alonso DT, Ayala PJ, López PM, Castillo LB. GÉRMENES NOSOCOMIALES MÁS FRECUENTES EN LA UNIDAD DE TERAPIA INTENSIVA. Rev Cub
Med Int Emerg. 2006. 5 (1);302-11.

11. Jover SA, Barcenilla GF, Barbé IE, García GM, López SR, Castellana PD, et al. INFECCIÓN NOSOCOMIAL POR GÉRMENES MULTIRRESISTENTES DURANTE 1 AÑO EN UN HOSPITAL DE SEGUNDO NIVEL. An Med Int. 2005. 22 (2); 59-64.

12. Hernández $\mathrm{OH}$, González $\mathrm{SN}$, Castañeda NJ, Arzate BP, Saldaña MC, Monroy DA, et al. INFECCIONES NOSOCOMIALES EN EL INSTITUTO NACIONAL DE PEDIATRÍA. Acta Pediatr Mex 2006. 27 (6); 325-8.

13. Briceño I, Suárez M. RESISTENCIA BACTERIANA EN LA UNIDAD DE CUIDADOS INTENSIVOS DEL HOSPITAL UNIVERSITARIO DE LOS ANDES. Medicrit 2006. 3 (2); 30-42. 14. Solano CA. INCIDENCIA DE INFECCIÓN NOSOCOMIAL EN UNA UNIDAD DE CUIDADOS INTENSIVOS. Disponible en: $h t t p: / / w w w . b i n a s s s$. sa.cr/revistas/rccm/v14n1-2/art2.pdf

15. Cardozo HG, De Colsa RA, Zepeda OG, Arzate BP, Gonzáles SN. MANIFESTACIONES CLÍNICAS Y FACTORES DE RIESGO PARA BACTERIEMIA POR BURKHOLDERIA CEPACIA EN NIÑOS. Rev Enf Inf Pediatr 2007. 20 (80); 92-98.

16. Quintanilla, Chanez J, Orellana VR, Alfaro CC. PERFIL MICROBIOLÓGICO DE INFECCCIONES NOSOCOMIALES EN LA UNIDAD DE TERAPIA INTENSIVA DEL HOSPITAL CLÍNICO VIEDMA. Gac Med Bol 2011. 34(1); 37-39.

17. Luna C, Gherardi C, Famiglietti A, Vay C. RESISTENCIA BACTERIANA Y ANTIBIÓTICO TERAPIA EN MEDICINA RESPIRATORIA Y TERAPIA INTENSIVA. Medicina 2001. 61 (5); 603-13.

18. Robles J, Ramírez L, Xicotencatl A, Vladez $M$, Chan F. INFECCIONES NOSOCOMIALES EN LA UNIDAD DE TERAPIA INTENSIVA PEDIÁTRICA. Falcón E. Temas selectos en Terapia Intensiva Pediátrica 2013. Vol 2. México. Ed Alfil. 1049-67.

19. Herrera-UM, Pérez-PP, Vásquez-VM, Santana-LV. PROFESIONALES DE LA SALUD ANTE LA MEJORA DE LA HIGIENE DE LAS MANOS: ESTRATEGIAS CLÁSICAS VERSUS ESTRATEGIAS AVANZADAS. Rev Chilena Infectol 2014. 31 (5); 534-41. 